\title{
UNDE DEN VRIDE MACHTEN WIDER IN DEM LANDE - KRIEGSZIELE IN DER HISTORIOGRAPHIE DES DEUTSCHEN ORDENS
}

\section{Prolegomena}

an fängt keinen Krieg an“, schreibt Clausewitz (1780-1831) in den
Skizzen zum unvollendeten achten Buch seines epochalen Werkes,
„oder man sollte vernünftigerweise keinen anfangen, ohne sich zu sagen, was man mit, und was man in demselben erreichen will; das Erstere ist der Zweck, das Andere das Ziel. Durch diesen Hauptgedanken werden alle Richtungen gegeben, der Umfang der Mittel, das Maß der Energie bestimmt; er äußert seinen Einfluss bis in die kleinsten Glieder der Handlung hinab“.

Diese - aus mediävistischer Sicht - moderne und rationalisierte Definition des Krieges wird man nicht ohne Brüche auf einen mittelalterlichen Ritterorden übertragen können - schon gar nicht, wenn dessen Geschichte einen so massiven Bruch aufweist wie die Abkehr vom Heiligen Land und dem Königreich Jerusalem und die Hinwendung zur selbst verantworteten Territorialisierung im südlichen Ostseeraum.

Diese beiden Phasen der mittelalterlichen Deutschordensgeschichte sind in der Chronistik des Ordens auf eigentümliche Weise getrennt. Deutschordensgeschichte wurde mit Peter von Dusburg zur preußischen Landesgeschichte: Diese vor der gesamten Geschichte des Ordens verwunderliche Neudefinition setzte in gewisser Weise die Auslöschung der bisherigen Memoria des Ordens voraus. Dass dies im Zusammenhang mit der umstrittenen Verlegung des Hochmeistersitzes auf die Marienburg stand, ist evident. Doch alle Details dieser Frage sind unklar.

Das Fehlen jeglicher innerer Memoria vor dem Auftreten des Ordens in Preußens zwingt zur kursorischen Behandlung der Frühzeit. Dass sie selbst für die preußischen und litauischen Kriege prägend wirkte, garantierten die dem Orden

1 C. v. Clausewitz, Vom Kriege, Bd. 3, Berlin ${ }^{3} 1869$, S. 69. 
bei seiner Gründung vorgegebenen Statuten, die er zwar fortschrieb, aber während des Mittelalters nicht von Grund auf erneuerte. Sie wurden im Umfeld der Kurie aus den Regeln der Templer und Johanniter synthetisiert. Dementsprechend banden sie den neu zu gründenden Orden mit einem doppelten Auftrag an das Heilige Land. Neben der Überwindung der Heiden durch militärisch-aggressive Operationen, war als Kriegszweck - der in der Diskussion um die „Kreuzzugsideologie “2 oft übersehen wird - die Neugestaltung der Territorien durch Übernahme sakraler und administrativer Aufgaben zu verrichten. So steht es ganz klar im Prolog der Deutschordensregel: Mit Gottes Hilfe haben die Makkabäer ${ }^{3}$ die Heiden uberwunden und vertilgeten, daz si die heiligen stete wider gereinigeten, die sie heten geunreint, unde den vride macheten wider in dem lande. ${ }^{4}$ Das Kriegsziel einer Niederwerfung des Feindes (so heißt das bei Clausewitz) dient dem Kriegszweck einer sakral gemeinten restauratio pacis - eine spätantike Formel für das Wohlergehen des Reiches - und die Wiederherstellung des (christlichen) Kultes.

Die Sakralität eines derart mit Waffengewalt erzwungenen Friedens ist zweifelhaft. Durch die Proklamation eines solchen Fernziels lässt sich leicht dauerhaftes aggressives Verhalten rechtfertigen. Doch sind echte Friedensperioden in der mittelalterlichen Geschichte - zumal in Palästina oder im Ostseeraum - die Ausnahme, so dass der in solchen Zwecksetzungen enthaltenen Utopie eines „ewigen Friedens" vielleicht doch mehr Aufmerksamkeit gewidmet werden sollte als es die Forschung bislang zugelassen hat. Das Konstrukt der „Kreuzzugsideologie“ ist Teil der Gottes- bzw. Landfriedenbewegung, aus der heraus schon der Kreuzzugsaufruf Urbans II. und die Proklamation einer Militia Nova durch den Hl. Bernhard verstanden werden müssen: Das für die europäischen Territorien verheerende Agressionspotential der Landfehden sollte zum Nutzen der Christenheit nach außen abgelenkt werden. Der zur Präfiguration des miles christianus herangezogene Judas Makkabäus war dem Mittelalter nicht der martialische conquering hero, den Händel in seinem Oratorium ${ }^{5}$ feiert: Mehr noch als seine militärischen Erfolge fas-

2 J. Trupinda, Ideologia krucjatowa w kronice Piotra z Dusburga (Peribalticum Meridionale 1), Gdańsk 1999.

3 Zur voraufgehenden Rezeption der jüdischen Befreiungskämpfer im Okzident vgl. S. Gougenheim, Les Maccabées, modèles des guerriers chrétiens des origines au XIIe siècle, Cahiers de civilisation médiévale 54 (2011), S. 3-20.

4 Die Statuten des Deutschen Ordens. Nach den ältesten Handschriften, hrsg. v. M. Perlbach, Halle/ /Saale 1890, S. 25.

5 Diese Chorpartie - in Deutschland als Weihnachtschoral mit dem später unterlegten Text Tochter Sion freue dich verbreitet - bezog sich ursprünglich auf Josua (HWV 64) und wurde 1751 von Händel nachträglich in das Makkabäeroratorium (HWV 63) eingeschoben. Es wurde zur Feier von William Augustus Duke of Cumberland (1721-1765) gestiftet, der allerdings als Sieger über die letzten schottischen Freiheitskämpfer (Schlacht von Culloden 1746) eher das 
zinierte die Neueinweihung des von Heliodor geschändeten Tempels im Jahr 164 vor Christus. ${ }^{6}$ Was in der jüdischen Tradition durch das nun wahrlich nicht kriegerische Chanukka-Fest ${ }^{7}$ vergegenwärtigt wird, war im christlichen Kontext eine Vorausdeutung auf die Vertreibung der Händler aus dem Tempel und auf das gereinigte Neue Jerusalem. Darum erklärt die Narratio de primordiis zum Kriegsziel: „dass nach erfolgter Rückgabe des Heiligen Landes an den christlichen Kultus in der heiligen Stadt Jerusalem errichtet werde die domus ordinis principalis (das Haupthaus des Ordens), gleichermaßen die Mutter, das Haupt und die Herrin."

\section{PRÄFIgURATIONEN}

Neben diesem triumphalen Gestus gab die im Alten Testament verschriftlichte Geschichtserfahrung des Volkes Israel genügend Anlass zur Skepsis und zur ethischen Überformung. Der Sieg über die Feinde kam nicht automatisch, sondern musste durch ein gottgefälliges Leben verdient werden. Die Bibelrezeption der Ritterorden konzentrierte sich gerade auf solche alttestamentarischen Werke, die den Glauben an die Waffen als eitel herausstellen. So wurden wahrscheinlich auch im Deutschen Orden schon in der Frühzeit durch Kreuzzugsprediger die Psalmen 105 und 106 als Rechtfertigung des militärischen und administrativen Handelns im Heidengebiet entdeckt. Literarisch manifestiert sich dies im Deutschordenskontext, soweit ich sehe, erst spät, nämlich in der so genannten Älteren Hochmeisterchronik, wo sie ein (möglicherweise aus einem älteren Text inseriertes) Lob auf Winrich von Kniprode präfigurieren. ${ }^{9}$ Ps. 105 beschreibt die Landnahme der Hebräer in Palästina und die Unterwerfung - eigentlich: Versklavung - der dort ansässigen Heiden, Ps. 106 den drohenden Verlust des gelobten Landes bei Abkehr von Gottes Geboten. Hier die beiden zentralen Passagen im Wortlaut der Vulgata:

Gegenbild zu Judas Makkabäus abgab, vgl.: Das Händel-Handbuch. 5. Händels Instrumentalmusik, hrsg. v. H. J. Marx, Laaber 2008, S. 197; R. A. Streatfeild, Handel, New York 1909, S. $314 \mathrm{f}$.

6 Vgl. I Makk 4, 36-61.

7 S. Galley, Das jüdische Jahr. Feste, Gedenk- und Feiertage (Beck'sche Reihe 1523), München 2003, S. 101-108.

8 Ut terra sancta christiano cultui restituta in civitate sancta Jerusalem domus fieret ejusdem ordinis principalis, mater caput pariter et magistra; zitiert nach: Scriptores rerum Prussicarum. Die Geschichtsquellen der preußischen Vorzeit bis zum Untergange der Ordensherrschaft, hrsg. v. Th. Hirsch, M. Toeppen, E. Strehlke, Bd. 1, Leipzig 1861, S. 221.

9 Vgl. unten Anm. 76. Der Text wird aus Predigten oder Admonitiones der Ordenskapitel geformt worden sein. 
Ps. $105 .{ }^{43}$ Et eduxit populum suum in exsultatione, electos suos in laetitia. ${ }^{44}$ Et dedit illis regiones gentium, et labores populorum possederunt, ${ }^{45}$ ut custodiant iustifcationes eius et leges eius servent. ${ }^{10}$

Ps. $106 .{ }^{39}$ et contaminati sunt in operibus suis et fornicati sunt in adinventionibus suis. ${ }^{40}$ Et exarsit ira Dominus in populum suum et abominatus est hereditatem suam $^{41}$ et tradidit eos in manus gentium, et dominati sunt eorum, qui oderunt eos. ${ }^{42}$ Et tribulaverunt eos inimici eorum, et humiliati sunt sub manibus eorum. ${ }^{43}$ Saepe liberavit eos; ipsi autem exacerbaverunt eum in consilio suo et corruerunt in iniquitatibus suis. ${ }^{11}$

Die Utopie des friedlichen und heiligen Landes wird mit massiver Aggression gewonnen. Bemerkenswert deutlich sagt Ps 105,44, dass Enteignung und Zwangsarbeit gottgewolltes Recht sind. Der Terminus regiones gentium - „Gebiete der Heiden" - macht für den mittelalterlichen Rezipienten das Ganze zum Modellfall für jedes Kreuzzugsunternehmen. Das wiederum traf sich vorzüglich mit der zeitgenössischen Rechtfertigung der Sklaverei. ${ }^{12}$

Die Möglichkeit zu materieller Ausbeutung durch Plünderungen und Tributforderungen, Erpressung von Lösegeldern und Heranziehung der Gefangenen zur Zwangsarbeit gehörten nicht nur in alttestamentarischer Zeit zu den wirtschaftlichen Vorteilen eines gewonnenen Krieges (wobei sich das Glück, wie im Falle der babylonischen Gefangenschaft, auch gegen den früheren Sieger kehren konnte). Fehden waren also nicht nur eine Frage der Ehrverletzung, sondern auch ein Wirtschaftsfaktor, den die Gottes- und Landfriedensbewegung ebenso zu unterbinden suchte wie das städtische Recht, etwa wenn im Magdeburger Recht die verwüstende Heimsuchung zum Hochgerichtsfall erklärt wird. ${ }^{13}$ Der Hilferuf Konrads von Masowien an den Deutschen Orden war eine Verzweiflungstat an-

10 Lutherbibel (1912): ${ }^{43}$ Also führte er sein Volk in Freuden und seine Auserwählten in Wonne ${ }^{44}$ und gab ihnen die Länder der Heiden, daß sie die Güter der Völker einnahmen, ${ }^{45}$ auf daß sie halten sollten seine Rechte und sein Gesetze bewahren.

11 Lutherbibel (1912): ${ }^{39}$ und verunreinigten sich mit ibren Werken und wurden abgöttisch mit ihrem Tun ${ }^{40}$ Da ergrimmte der Zorn des HERRN über sein Volk, und er gewann einen Greuel an seinem Erbe ${ }^{41}$ und gab sie in die Hände der Heiden, daß über sie herrschten, die ihnen gram waren. ${ }^{42}$ Und ibre Feinde ängsteten sie; und sie wurden gedemütigt unter ihre Hände. ${ }^{43}$ Er errettete sie oftmals; aber sie erzürnten ihn mit ihrem Vornehmen und wurden wenig um ihrer Missetat willen.

12 S. Ekdahl, Die Christianisierung Litauens als Dilemma des Deutschen Ordens, in: Lietuvos krikscionejimas Vidurio Europos kontekste, hrsg. v. Vydas Dolinskas, Vilnius 2005, S. 189-205, hier S. 194 unter Berufung auf J. Fried, Über den Universalismus der Freiheit im Mittelalter, Historische Zeitschrift 240 (1985), S. 313-361, hier S. 315 f. Zum Thema allgemein: Unfreie Arbeits- und Lebensverhältnisse von der Antike bis in die Gegenwart: eine Einführung (Sklaverei, Knechtschaft, Zwangsarbeit 1), hrsg. v. E. Herrmann-Otto, Hildesheim u. a. 2005.

13 H. Holzhauer, Heimsuchung, in: Lexikon des Mittelalters, Bd. 4, München 2004, Sp. 2036. 
gesichts der verheerenden räuberischen Übergriffe der prusischen Nachbarn. „Der Deutsche Orden war kein Mönchsorden, der die Heiden durch Missionierung zum katholischen Glauben bekehren sollte, er war ein geistlicher Ritterorden mit einem Kampfauftrag, dessen Sinn es war, die von den Ungläubigen bedrohten Christen und unter ihnen die Neugetauften, die Neophyten, zu beschützen “. ${ }^{14}$ Die Nachhaltigkeit seines Erfolges beruhte aber darauf, dass er mit seiner Landnahme auch für die Prussen das Kirchenrecht (und damit den Gottesfrieden) bzw. durch die Verbreitung des Kulmer Rechts die städtische Gerichtsbarkeit durchsetzte. Allerdings war es illusionäre Verkennung, wenn man diesen zivilisatorischen Schritt wirtschaftlich alleine auf den Fleiß der aus dem Reich ins Land geholten Siedler zurückführte, zumal deren Zustrom infolge des Schwarzen Tods bald aussetzte. Ausbeutung und Zwangsarbeit wanderten mit den Heerzügen, ihre Vermeidung (oder wenigstens Milderung) waren das stärkste Motiv der "Schwertmission“. Getaufte Prussen konnten am Wohlstand der neuen Administration teilhaben. Während man sich im Westen und Süden (also gegenüber Pommern und Polen) mit finanziellen Transaktionen neue Wirtschaftsregionen erschloss, geschah dies nach Osten in traditioneller Weise durch Gewalt. Der Friede im Lande wurde finanziert (und war nur finanzierbar) durch die fortgesetzte Aggression gegenüber den litauischen Nachbarn.

Aus dieser Spannung erwuchs die janusköpfige Rolle des Deutschen Ordens im Ostseeraum. ${ }^{15}$ Sie bestimmte die Körperschaft nach außen wie nach innen.

\section{Die Programmatik der Novi MaCchabaEI}

Der Ansatzpunkt der Deutschordens-Chronistik ist von den Grundlagen her durch und durch mittelalterlich. Der deutsche Orden war als geistliche Körperschaft im Sinne der militia nova des Heiligen Bernhard, eyne muer [...] vor dy cristinlant weder dy heydin. ${ }^{16}$ Das ist universell gemeint. In der Praxis aber bezog der Orden seinen Auftrag jeweils auf ein konkretes Territorium, zunächst Palästina, dann aber Preußen und Livland, und er setzte sich energisch zur Wehr, wenn man ihn mit der Universalität seiner Ritterschefte konfrontierte und z. B. gegen andere Heiden - wie die Türken - einsetzen wollte.

14 Ekdahl, Christianisierung (wie Anm. 12), S. 191.

15 Ebd., S. 197 f.

16 So die Posilge-Chronik, in: Scriptores rerum Prussicarum (wie Anm. 8), Bd. 3, Leipzig 1866, S. 13-399, hier 340 . 
Demnach kann man eine erste Definition wagen. War also der Kriegszweck der Heidenkampf, so wurde für die Ritterorden in Palästina das Kriegsziel viel konkreter, nämlich geographisch, umrissen. Für die Frühzeit des Deutschen Ordens in Palästina und für die Jahrzehnte bis zur Annektion Pommerellens 1309 mag das uneingeschränkt gelten. Danach wurde das Konzept verbal fortgeschrieben, ob es allerdings noch für eine Beschreibung der konkreten Kriegssituation hinreicht, bleibt zu prüfen.

Die Bindung an das Territorium stellt sich nicht bloß als eine Frage der militärischen Pragmatik dar, wie sie bei Clausewitz in den Gegensatz von absolutem und wirklichen Krieg gefasst wird ${ }^{17}$ sondern weist über den Krieg hinaus in den Bereich der spirituellen wie der wirtschaftlichen Fundierung. Als Bonifaz IX. 1403 die Litauenfahrten verbot, zeigte sich Konrad von Jungingen unbeeindruckt und zur Fortsetzung der reysen entschlossen..$^{18}$ Denn aus dem Schutzauftrag gegen heidnische Angriffe und Racheakte erwuchsen, je länger die eigentliche Bekehrung zurücklag, ganz andere, nämlich administrative, Aufgaben, die dem Deutschen Orden ein anderes Gesicht verliehen. Als administrative Körperschaft aber trat er in offene Konkurrenz zu den Potentaten, ja den Herrschaftssystemen in den angrenzenden Staaten und mehr und mehr auch im eigenen Land. Die Chronistik des Deutschen Ordens zielt sogar - und es werden weitere Studien erforderlich sein, um das auszuloten - auf eine unlösbare, weil sakrale, Bindung der Körperschaft an das lant, das ihm als von Gott verliehen erscheint. ${ }^{19}$ Angelpunkt dieses Verständnisses könnte der Psalm 105 gewesen sein.

Wenn so die Ordensherrschaft in Preußen durch die hebräische Landnahme in Palästina präfiguriert wird, so ist vor dem Jüngsten Gericht kein Ende der Ordensherrschaft im Lande denkbar, selbst wenn sich aktuell kein Heide in ihm aufhält oder es bedroht.

In den Frühzeit des Ordens ist eine solche Bezugnahme fast selbstverständlich.

17 A. Nikžentaitis, Prisoners of War in Lithuania and the Teutonic Order State (1283-1409), in: Der Deutsche Orden in der Zeit der Kalmarer Union 1397-1521 (Ordines militares. Colloquia Torunensia Historica X), hrsg. v. Z. H. Nowak unter Mitarbeit v. R. Czaja, Toruń 1999, S. 193-208, S. 195, unterscheidet zwischen "practical aims (the conquest of Lithuania)“ und dem ,ideological task (the baptism of Lithuania)“, die in Einklang zu bringen waren: ,the Order's fourteenth century contemporaries gained the impression that the Order's aim was not only to conquer Lithuania but also to baptise her".

18 Ekdahl, Christianisierung (wie Anm. 12), S. 197.

19 Diese Bindung scheint proportional zur administrativen Rolle des Ordens zu stehen: Sie ist in Preußen deutlicher ausgeprägt als in Livland, wo die Landesherrschaft weniger ausgeprägt war. 
Noch deutlicher als die eingangs angeführte Narratio de primordiis stellt Peter von Dusburg ${ }^{20}$ nach erneuter Berufung auf die Makkabäer auch Preußen als Aufgabengebiet heraus: Hec est causa, propter quam fratres domus Theutonice ab introitu terre Prvssie usque ad presentem diem utuntur gladiis continue, ut in promptu habeant, quo se defendant, si contra eos ab hostibus insurgeret aperta impugnacio vel occulta. Eodem modo utimur armis virtutum contra apertas impugnaciones dyaboli. ${ }^{21}$

Peter wechselt hier von der militärischen Argumentation auf die spirituelle. Sein Erzählen vom Kriege ist stets ein Aufruf aus dem Blickwinkel der monastischen Theologie. Für ihn ist der körperliche Kampf zwar kein Akzidenz, aber dem essentiellen geistigen Kampf stets untergeordnet. Die "Schwertmission“ wird in ihrer Rechtfertigung nicht angetastet, aber mit hohen moralischen Anforderungen für jeden verbunden, der in ihrem Namen agieren will. Wirtschaftliche Aspekte werden erst gar nicht thematisiert.

Mit dem Verlust von Akkon, spätestens aber mit der Verlegung des Hochmeistersitzes nach Marienburg beginnt für Peter von Dusburg die eigentliche Geschichte des Deutschen Ordens. Das Land, das Gott ihm geben wird, ist nicht Palästina und nicht das Burzenland, und wenn er nun mit kaiserlichem und päpstlichem Segen seine - wie es die Statuten nennen - ritterschefte nach Preußen transferiert, so muss er sich dieser unausweichlichen Verpflichtung stellen.

Wohlgemerkt: Es geht um das Land, nicht um seine Bewohner. Es geht um den Glauben, nicht um die Gläubigen. Diese Ausrichtung lassen die Texte, die wir „Deutschordenschroniken“ nennen, schon in ihrer Selbstbezeichnung erkennen. Peter von Dusburg schreibt das „Chronicon terrae Prusiae“, und Nikolaus von Jeroschin dementsprechend die Kronike von Pruzinlant, wir haben kein Chronicon ordinis theutonicorum und keine „Kronike vom Dutschen Huse“ [sind die Eintragungen richtig? - bitte vereinheitlichen Kursive und „”, Titel eigentlich kursiv]. Ihre Berichte stellen zwar durch Übernahme der Narratio deprimordiis den Bezug zur Gründung des Ordens im Heiligen Land her, doch schweigen sie über die Geschichte, berichten weder über die Besitztümer und Burgen noch die Heldenta-

20 David hostes regni sui devicit, et Machabei civitatem sanctam Jerusalem destructam reparaverunt, et templum domini sordibus gencium pollutum interfectis hostibus mundaverunt. Ofortissimi milites et bellatores incliti, induite hec arma et vindicate injuriam crucifixi domini et terram sanctam Cristianis debitam recuperate, ab infidelibus occupatam. Confortamini et non dissolvantur manus vestre in bello, gl. Peter von Dusburg, Cronica terre Prussie, hrsg. v. M. Töppen, in: Scriptores rerum Prussicarum (wie Anm. 8), Bd. 1, S. 44; Neuedition: Piotr z Dusburga, Kronika ziemi Pruskiej (Monumenta Poloniae Historica, nova series XIII), hrsg. v. J. Wenta, S. Wyszomirski, Kraków 2007, S. 41.

21 Scriptores rerum Prussicarum (wie Anm. 8), Bd. 1, S. 45 (= Monumenta Poloniae Historica, nova series 13 (wie Anm. 20), S. 43). 
ten des Ordens in Palästina. Wer darüber lesen will, muss zu ordensfremden Texten greifen, etwa der Chronik der vier Orden von Jerusalem ${ }^{22}$ oder Ottokars Steirischer Reimchronik. Das ist keineswegs Zufall, sondern zielgerichtete Programmatik: die ältere Bindung an das Heilige Land wird überdeckt durch die neue an die terra Prusiae $^{23}$, ex silentio ein Bekenntnis zur Verlegung des Hochmeistersitzes auf die Marienburg, weitab vom Mittelmeer.

Gegenüber den traditionellen Kreuzfahrerchroniken macht Peter von Dusburg etwas sehr Folgenreiches. Albert von Aachen oder Fulbert von Chartres erklären ihren Lesern nicht, gegen was für Ungläubige sich der Kreuzzug richtete. Es waren Heiden, die die heiligen Stätten schänden und Pilger misshandeln. Das reichte den Chronisten. ${ }^{24}$ Peter stellt - möglicherweise in der Nachfolge des Wilhelm von Tyrus, der als Landeskind eine persönliche Bindung an Palästina hatte uns die Gegner vor: und zwar mit größerer Korrektheit, als Wilhelm das für den Islam tat. Peters Nachrichten sind bis heute eine zentrale Quelle über Religion und Kult der Prussen; Nikolaus von Jeroschin und die späteren Chronisten der Ordenszeit haben dem kaum etwas hinzuzufügen, aber sie lassen es auch nicht aus.

Das bedeutet nicht, dass diese Texte sich nicht mit der Lebensform und der Legitimation des Ordens befassen würden. Diese Bereiche sind m. E. sogar der Ausgangspunkt. Als Vorstufe von Peters Chronik vermute ich sowohl für die grundsätzliche Exposition de armis carnalibus et spiritualibus als auch für die erzählenden Bereiche jene Ermahnungen, die von Priesterbrüdern zu Beginn der wöchentlichen Ordenskapitel gehalten wurden. Sie schlossen natürlich auch Exempla aus der Ordensgeschichte ein. Die Botschaft ist immer: Wenn dem äußeren Krieg nicht der innere entspricht, wenn also dem adligen Kampf nicht der asketische zur Seite tritt, war alles umsonst. Das Erzählen vom Krieg wird transformiert durch das Postulat der Spiritualität. Es bereitet den Kämpfer damit für andere Aufgaben vor.

Nikolaus von Jeroschin macht das gegenüber seiner lateinischen Vorlage sogar noch deutlicher. Er lässt mit dem vagen Quellenhinweis hân ich gelesin (V. 4655) einem Bruder aus Rehden, der in einen strengeren Orden wechseln wollte, ein Traumgesicht zuteil werden. Es erscheinen ihm die Repräsentanten der großen

22 Zur dieser nur in einer Handschrift des Deutschordenszentralarchivs in Wien überlieferten Chronik vgl. U. Arnold, Die vier Orden von Jerusalem, in: Verfasserlexikon. Die deutsche Literatur des Mittelalters, 2. Aufl., Bd. 1, Berlin u. a. 1978, Sp. $1261 \mathrm{f}$.

$23 \mathrm{Zu}$ Lebzeiten Peters von Dusburg war es wohl noch nicht mit letzter Sicherheit ausgemacht, dass Preußen die Hauptaufgabe des Deutschen Ordens bleiben werde, auch wenn mit dem Tode Karls von Trier die Gegner dieser Orientierung ihre Galionsfigur verloren hatten.

24 H. Möhring, Heiliger Krieg und politische Programmatik. Salahadinus Tyrannus, Deutsches Archiv für Erforschung des Mittelalters 39 (1983), S. 417-466, hier S. 442 f. 
Orden: Bernhard von Clairvaux für die Zisterzienser, Dominicus und Franziskus für die Mendikanten, aber sie weisen den Bruder alle als unwürdig zurück. Danach erscheint die Gottesmutter mit einer Schar von wundenübersäten Deutschordensbrüdern und droht, ihn auch aus dem Deutschen Orden auszustoßen, den er so gering achte. Daraufhin bereut er vor dem Ordenskapitel und findet alsbald im Prussenkampf selbst den Märtyrertod (V. 4723-4744). Dieser asketischen Linie bleibt das historische Ordensschrifttum, auch in der eigentlich nüchternen Posilge-Chronik und noch in den allerletzten Reformentwürfen des 16. Jahrhunderts, immer treu. Die Verweltlichung des Ordens gefährdet (entsprechend der Drohung des Ps. 106 und anderer alttestamentarischer Stellen) seine Unbesiegbarkeit. Das erklärt die Ältere Hochmeisterchronik, das wird auch die Ermahnung des Kartäusers sagen, selbst Simon Grunau lässt die Verderbnis der preußischen Lande aus der Abkehr der Ordensritter vom asketischen Ideal kommen, die den von Gott zunächst geförderten Orden zu einem Werkzeug des Teufels gemacht habe. Solche ideologisierten Verkennungen haben wohl nicht unmaßgeblich dazu beigetragen, dass der Orden nach 1454 nur selten zu einer realistischen Einschätzung seiner wirtschaftlichen und militärischen Situation fand.

Exempla über die weltlichen und die geistigen Waffen werden auch in den Admonitiones der Ordenskapitel vor der preußischen Invasion verwendet worden sein. Außerhalb des Ordens, etwa in Ottokars Steirischer Reimchronik, ist einiges davon überliefert, im Orden selbst aber nicht. Auch die Vision in Rehden hat nichts spezifisch „Preußisches“, sie könnte ebenso gut über einen Ritterbruder in Palästina erzählt worden sein. Zweck einer solchen Erzählung ist ja auch nicht, die Zuhörer zum kollektiven Märtyrertum anzustiften, sondern darauf hinzuweisen, welchen bedeutenden Anteil der Deutsche Orden am Schatz der Kirche hat - so dass die ihm gewährten Privilegien und Ablässe zu recht bestehen und in dieser wie in der künftigen Welt belohnt wird, wer ihn durch Stiftungen fördert. Auch hier sind, wenngleich verborgen, die wirtschaftlichen Interessen nicht fern.

Bei Peter von Dusburg ist das Ziel der kriegerischen Unternehmungen noch leicht zu fassen. Das Land der Prussen soll in ein christliches Gebiet verwandelt werden, dessen Integrität nach innen wie nach außen vom Deutschen Orden garantiert wird. Doch gerade dadurch entsteht die komplexe Verbindung zwischen Land und Orden. Sie wird teilweise verständlicher, aber nur teilweise, wenn wir uns mit Percy Ernst Schramm und Theodor Mayer daran erinnern, dass das Mittelalter keinen Staatsbegriff kannte. ${ }^{25}$ Was wir „Deutschordensstaat“ nennen,

${ }^{25}$ Andererseits verstellt und vereinfacht diese etatistische Sicht die Verhältnisse, weil sie viel zu sehr auf den Hochmeister als administrativen Agenten abhebt und die Eigendynamik der geistlichen Korporation übergeht. 
ist für die Zeitgenossen entweder Preußenland oder Livland - im besten Fall beides zusammen; der Deutsche Orden darin jeweils die Schutzmacht. Erst in der Posilge-Chronik, die sich damit andern Zeugnissen aus dem pomesanischen Bistum zur Zeit Konrads von Jungingen einordnet, erscheint der Hochmeister nicht der Orden - auch als weltlicher Landesherr und Fürst. Die Repräsentanten des Landes heißen ohne Rücksicht auf ethnische oder korporative Unterschiede einfach Pruszen. Nur wenn es die Körperschaft selbst angeht, ist der ordin als solcher gekennzeichnet. Die Kriege führt, so möchten es diese Annalen suggerieren, das Land, unterstützt von den adligen Preußenfahrern, die einfach herrin heißen. Zweck aller kriegerischen Unternehmungen vor dem Großen Krieg mit Polen, ja selbst nach 1411, bleibt die Abwehr der - echten oder vermeintlichen Heidenüberfälle von diesem Land und die Reinigung dieses Landes vom Unglauben, so dass das gesicherte und geheiligte Land in Frieden leben kann.

Die Wirksamkeit dieser Konzepte zeigt sich in den Kanonisationsakten der Dorothea von Montan, also in der pragmatischen Schriftlichkeit. Dorothea, die als Schutzpatronin Preußens etabliert werden soll, schützt auf wundersame Weise den Hochmeister Konrad von Jungingen vor der Kriegslist der Litauer. Der Hochmeister gab dies ohne Vorladung sponte, libere et voluntarie ${ }^{26}$ als 21 . Zeuge zu Protokoll. Er habe quattuor pericula in reysa - vier gefährliche Situationen während eines Feldzuges gegen die Litauer im Jahr 1394 durch das Eingreifen der Rekluse im Dom zu Marienwerder überlebt. In höchster Bedrängnis habe der Hochmeister einen Brief des pomesanischen Bischofs Johannes erhalten, eamque sibi perlegifecit. In qua scriptum erat, quomodo mater Dorothea, dum in humanis ageret, de eo predixisset, quod in prima sua reysa, quam faceret contra Lituanos, haberet subire quatuor pericula, scilicet in mari, in quadam insula, ante quoddam castrum et in quodam nemore. Et si in suis quadrigis et balistariis et multitudine exercitus confderet, valde difficiliter evaderet; sed si se et suos omnipotententi Deo committeret, faciliter Die aiutorio evaderet. ${ }^{27}$

Es ist fast wie eine Geschichte aus der Chronik Peters von Dusburg, wo die Marienritter durch den Eingriff der Gottesmutter mehr als einmal aus der Hand der Feinde gerettet werden; zumal Petrus unter Verweis auf Judiths Sieg über Holophernes ausruft: quis in arcu suo speravit et gladius eius salvavit eum ${ }^{28} \mathrm{Konrad}$

26 Die Akten des Kanonisationsprozesses Dorotheas von Montau von 1394 bis 1521 (Forschungen und Quellen zur Kirchen- und Kulturgeschichte Ostdeutschlands 15), hrsg. v. R. Stachnik, Köln u. a. 1978, S. 64.

27 Stachnik, Akten (wie Anm. 26), S. 65.

28 Scriptores rerum Prussicarum (wie Anm. 8), Bd. 1, S. 40, 45 (= Monumenta Poloniae Historica, nova series 13 (wie Anm. 20), S. 34). 
versichert denn auch, dass er mit seinem Heere a dictis periculis fuit ex intercessione beate Dorothee liberatus. ${ }^{29}$

Bemerkenswert, dass trotz der Mitwirkung des pomesanischen Bischofs Johannes Mönch bei der Übermittlung von Dorotheens Schreiben und trotz des in Marienwerder abgehaltenen Kanonisationsprozesses die Chronik des Riesenburger Offizials davon nichts weiß. Die Posilge-Chronik beschreibt den Feldzug ausführlich und bezeichnet die mehrfache Errettung des Ordensheeres als eyn gros wunder, dorumb man got groslich lobin sal und dankin synen gnaden ${ }^{30}$, erwähnt aber Dorothea mit keinem Wort. ${ }^{31}$ Doch stellt auch sie Kriegsgeschehen und sakrale Sphäre eng nebeneinander.

\section{Geostrategische Konzepte?}

Die Parallelität von Krieg, Spiritualität und Wirtschaftsinteressen wirft ein besonderes Licht auf die als „Blütezeit“ des Ordens geltende zweite Hälfte des 14. Jahrhunderts - insbesondere auf Konrad von Jungingen, in dessen Person all dies zusammenzufließen scheint. Der Ordensstaat hatte seine größte flächenmäßige Ausdehnung erreicht. Sein Reichtum war legendär; allein durch Zukauf von teilweise dubiosen Erbansprüchen konnten ganze Landstriche erworben werden. Der Ausbau der Dome und der Marienburg dokumentierte ebenso wie die fortschreitende Trockenlegung der Sümpfe, dass der Segen der Gottesmutter und ihres Sohnes auf dem Lande ruhte. Für die Zeitgenossen war dies evident; moderne Betrachter haben es auf eine umsichtige und planvolle Strategie der Ordensleitung zurückgeführt.

Nimmt man die Karte des „Deutschordensstaates“ in einem beliebigen historischen Nachschlagewerk, so scheinen die militärischen und wirtschaftspolitischen Kriegsziele des Deutschen Ordens im 14. Jahrhundert rasch erfasst: Gewinnung einer durchgehenden Landverbindung zwischen den Ostgrenzen des Heiligen Römischen Reiches und dem äußersten Livland (Territorialisierung); Abdrängung aller konkurrierenden Territorialstaaten von der Ostsee und den Flussmündungen und Errichtung eines Handelsmonopols in der südlichen Ostsee: „Der Heidenkampf diente als ideologische Begründung; daneben gab es aber auch die unterschiedlichsten Motive, die, was den Orden selbst betrifft, häufig real-

29 Stachnik, Akten (wie Anm. 26), S. 65.

30 Scriptores rerum Prussicarum (wie Anm. 8), Bd. 3, S. 194-196, das Zitat auf S. 194.

31 Rein militärisch Wigand von Marburg, vgl. Scriptores rerum Prussicarum (wie Anm. 8), Bd. 2, Leipzig 1864, S. 654-662. 
politischer Art waren, ging es doch um die Erweiterung des Ordenslandes im Osten und die angestrebte territoriale Verbindung der livländischen und preußischen Ordenszweige über die Landbrücke Samaiten“.32

Mit dem Frieden von Sallinwerder 1398 schien für wenige Jahre dieses geostrategische Ziel erreicht - falls es denn als solches bewusst definiert war. ${ }^{33}$ Solche Vorstellungen bestimmten dennoch die moderne Deutschordens-Historiographie, die Johannes Voigt als spätem Zeitgenossen des Clausewitz begann. Allerdings sind hier Bedenken am Platz. Kein Hochmeister und kein Ordensmarschall verfügte jemals über eine Karte des „Deutschordensstaates“. Als Konrad von Jungingen für die Marienburg eine mappa mundi beschaffen und sie an einer Wand des Hochschlosses anbringen ließ, ${ }^{34}$ wurde dies wegen der Eigentümlichkeit des Vorfalls akribisch in den Akten des Tresslers notiert. ${ }^{35}$ Von Preußen wird darauf aber kaum mehr als die Lage im europäischen Norden, fernab von Jerusalem, erkennbar gewesen sein. Militärische oder wirtschaftliche Ziele waren mit der Karte sicher nicht verbunden, sondern der Wunsch nach Teilhabe an der Wissenskultur der Zeit. Die älteste bekannte Karte des Preußenlandes schuf Heinrich Petri ${ }^{36}$ in Basel für die Opera Omnia des Enea Silvio Piccolomini von 1551. Militärisch war sie unbrauchbar. Eine nach heutigen Maßstäben verwendbare Karte des Preußenlandes entwarf erst Kaspar Hennenberger im Jahr 1576 mit seiner bis ins 18. Jahrhundert nachgedruckten „preußischen Landtafel“. ${ }^{37}$

32 Ekdahl, Christianisierung (wie Anm. 12) S. 191.

33 "Geostrategie" ist ein Begriff aus der sich aufheizenden Welt des Imperialismus, der rasch zu einer Kontrastierung von „West" und „Ost" führte; man vgl. etwa H. J. Mackinder, The Geographical Pivot of History von 1904, in: ders., Democratic Ideals and Reality, Washington 1996, S. 175-194; zur Kritik P. Venier, The Geographical Pivot of History and Early $20^{\text {th }}$ Century Geopolitical Culture, Geographical Journal 170 (2004), S. 330-336; und den Sammelband Global Geostrategy. Mackinder and the Defence of the West, ed. B. W. Blouet, New York 2005.

34 A. Mentzel-Reuters, Arma spiritualia. Bibliotheken, Bücher und Bildung im Deutschen Orden (Beiträge zum Buch- und Bibliothekswesen 47), Wiesbaden 2003, S. 29.

35 Das Marienburger Tresslerbuch der Jahre 1399-1409, hrsg. v. E. Joachim, Königsberg 1896, S. 62.16-25

36 Zur Person: H. Grimm, Henricpetri, Heinrich von, in: Neue Deutsche Biographie, Bd. 8, Berlin 1969, S. $552 \mathrm{f}$.

37 Als Erläuterung erschien: K. Hennenberger, Kurtze und warhaffige Beschreibung des Landes zu Preussen, Königsperg 1584, in die u. a. eine Kurzfassung der irrtümlich Bartholomaeus Wartzmann zugeschriebenen preußischen Landeschronik einfloß, vgl. A. Mentzel-Reuters, Von der Ordenschronik zur Landesgeschichte. Die Herausbildung der altpreußischen Landeshistoriographie im 16. Jahrhundert, in: Kulturgeschichte Ostpreußens in der Frühen Neuzeit, hrsg. v. K. Garber, M. Komorowski, A. E. Walter (Frühe Neuzeit 56), Tübingen 2001, S. 581-637, hier 636 f. mit weiterführender Literatur. 
Im Kartenmaterial spiegelte sich also kein strategisches Konzept des Deutschen Ordens. So hat die Forschung in Ermangelung von echten Kriegstagebüchern der Heeresleitung versucht, die Chroniken in diesem Sinne zu lesen. Dem ist energisch zu widersprechen. Es handelt sich hier um erzählende Quellen, ${ }^{38}$ die nach literarischen und nicht nach generalstabmässigen Gesichtspunkten verfasst wurden.

\section{Die Posilge-Chronik und ihre Programmatik}

Somit sind wir bei den Annalen, die gemeinhin mit dem Namen des Johann von Posilge verbunden werden. ${ }^{39} \mathrm{Ihr}$ Berichtszeitraum ist 1360 bis 1419. Die Feldzüge, über die sie handeln, spielten sich vor allem in Samaiten ab. Die Chronik findet ihren feierlichen Höhepunkt mit dem Vertrag von Sallinwerder (1398) und der Taufe der Bojaren von Samaiten am 9. Januar 1401 auf der Marienburg. Diese Ereignisse schienen die Garanten eines „ewigen Friedens“, für den - zumindest nach dem Annalisten der Posilge-Chronik - das Generalkapitel des Jahres 1400 das Fest Mariä Heimsuchung in der Ordensliturgie heraufstufte und verfügte, das man uf allin husern des ordens noch der frumesse sal luten pro pace eyne glocke und sprechin dry pater noster und dry Ave Maria, uf das unser here uns behalde in syme frede; dorczu der pabist grosin applas und gnade hat gegebin. ${ }^{40}$

An dieser Stelle schloss nach meinem Dafürhaltung die ursprüngliche lateinische Chronik; ungünstige Entwicklungen, aber auch das Bemühen, den Frieden von Sallinwerder zu retten, dürften dazu geführt haben, dass man sich an eine deutsche Bearbeitung und eine deutsche Fortschreibung machte.

Bei der Posilge-Chronik hat sich die Forschung mehr mit der Autorenfrage beschäftigt als mit dem Inhalt und der Programmatik der Chronik. In der Intitulatio der um $1420 \mathrm{zu}$ datierenden Berliner Handschrift Ms. Boruss. $2^{\circ} 241$ hören wir, dass her Johannes, officialis von Resinburg die Chroniken habe beschrebin zcu

38 Zur Typologie des Begriffs vgl. E. Boshof, K. Düwell, H. Kloft, Grundlagen des Studiums der Geschichte. Eine Einführung (Böhlau-Studien-Bücher. Grundlagen des Studiums), Köln ${ }^{5} 1997$, S. 116-127.

39 Die ältere Forschung zusammenfassend U. Arnold, Johann von Posilge, in: Verfasserlexikon. Die deutsche Literatur des Mittelalters, 2. Aufl., Bd. 4, Berlin 1983, Sp. 710 f. Neuere Ansätze bei J. Wenta, Verfasserschaft der sog. Chronik des Johann von Posilge, Preußenland 28 (1990), 1, S. 1-9; R. G. Päsler, Deutschsprachige Sachliteratur im Preußenland bis 1500. Untersuchungen $z u$ ihrer Überlieferung (Aus Archiven, Bibliotheken und Museen Mittel- und Osteuropas 2), Köln 2003, S. 284-290.

40 Scriptores rerum Prussicarum (wie Anm. 8), Bd. 3, S. 240. 
Latino, unde wurdin gewandelt dornoch yn das Dutsche unde vortan beschrebin noch syme tode. ${ }^{41}$

Johannes Voigt hat unter Heranziehung der Urkundenüberlieferung daraus den Offizial Johann von Posilge als Autor erschlossen. Nach dem Zeugnis von Simon Grunau, dem berüchtigten „Lügenmönch aus Tolkemit“" ${ }^{42}$ lautete der Name des Autors aber Johannes Lindenblatt. Voigt wollte das nicht ganz verwerfen und ließ den Namen auf dem Titelblatt seiner editio princeps stehen. ${ }^{43}$ Das wurde von übereifrigen Gelehrten übernommen und auch in die mittelalterlichen Handschriften hineingepinselt, ${ }^{44}$ was die Verwirrung erhöhte, aber nicht die Glaubwürdigkeit. Ernst Strehlke, der 1866 die bis heute maßgebliche Edition in den Scriptores rerum Prussicarum vorlegte, verwarf den Namen „Lindenblatt“ ohne Weiteres und erwog für wenige Zeilen einen späteren Offizial namens Johannes, nämlich Johann von Reddin, hatte sich aber längst, wie zuvor Voigt, auf Johannes von Posilge festgelegt. Vor nunmehr zwanzig Jahren hat Jarosław Wenta den Johann aus Rehden wieder aufgegriffen. Ralf Päsler verwarf in der Überprüfung dieser Thesen alle beiden Offiziale und beschränkte sich auf einen nicht fassbaren "Johann, Offizial von Riesenburg“, einen Verfasser namens "Johann Lindenblatt" hielt er immerhin wieder für denkbar. ${ }^{45}$

Die Lösung sehe ich darin, dass die Intitulatio von Ms. Boruss. $2^{\circ} 241$ eine vor 1411 verfasste Verfasserangabe der überarbeiteten lateinischen Vorlage nachbildete und ohne Plausibilitätsprüfung hinzusetzte, dass die eine deutsche Bearbeitung und Fortführung post mortem - natürlich des Posilge - erfolgte.

Die Geschichte der Chronik lässt sich dann wie folgt rekonstruieren. Johann von Posilge setzte die für uns verlorenen lateinischen Annales Resinburgenses auf, die zu einem erheblichen Teil - nämlich mindestens bis 1393 - eine Bearbeitung des Annalista Thorunensis waren. Daneben wurde von ihm eine Hauschronik der pomesanischen Kanzlei herangezogen, die über die Jahre nach einem stereotypen

41 Ebd., S. 79.

42 Dieses bei Nennung von Grunaus Namens unvermeidliche Epitaph geht letztlich auf Johannes Voigt zurück, vgl. J. Voigt: Geschichte Preussens von den aeltesten Zeiten bis zum Untergange der Herrschaft des deutschen Ordens, Bd. 2, Königsberg 1827, S. 523 f., Anm. 2 und öfter.

43 Es handelt sich bei Grunau möglicherweise um eine Verwechslung mit dem Danziger Chronisten Johannes Lindau, vgl. Mentzel-Reuters, Von der Ordenschronik zur Landesgeschichte (wie Anm. 37), S. $612 \mathrm{f}$.

44 Päsler, Sachliteratur (wie Anm. 39), S. 104 nach SB PK Berlin, Ms. Boruss. 2² 241 ohne Angabe einer Datierung. Ebenso ins 18. Jh. (1713) gehört die Abschrift der Chronik Dresden, Landesbibliothek, Mscr. G 38, auf deren Kolophon Päsler die Widerlgung von Wentas Zuweisung der Chronik an Johann von Reddin baut, vgl. Päsler, Sachliteratur (wie Anm. 39) S. 287 f.

45 Päsler, Sachliteratur (wie Anm. 39), S. 289. Theoretisch könnte dies den Bearbeiter der deutschen Textfassung bezeichnen. Mir scheint jedoch die Quellenlage keineswegs sicher. 
Schema gewachsen war und wohl mehr als einen Verfasser hatte. Aus ihr stammen die jährlichen Rubriken: (1) zum Wetter, (2) zu den Litauenfahrten, (3) Memorabilien allgemeiner Art, etwa der Einbruch von Epidemien und Besetzungen hoher kirchlicher Ämter.

Johann von Posilge gab das Offizialat 1406 auf und verstarb 1409. Einen Zusammenhang zwischen Offizialat und den historiographischen Notizen scheint es nicht zu geben. Für Johann von Posilge konnte allenfalls ein allgemeines landesgeschichtliches Interesse glaubhaft gemacht werden. ${ }^{46}$ Sein Manuskript verblieb in den Amtsräumen des Riesenburger Schlosses. Die Intitulatio der Berliner Handschrift Ms. Boruss. $2^{\circ} 241$ beschreibt exakt den Zustand, der zwischen Posilges Tod (1405) und vor der Ernennung Johanns von Reddin zum Offizial (1411) bestand. Die Bezeichnung her Johannes, officialis von Resinburg ist zu diesem Zeitpunkt noch eindeutig: Es hatte bislang nur einen pomesanischen Offizial mit Namen Johann gegeben (nämlich Johann von Posilge). Diesem schreibt die Intitulatio aber nur die (heute verlorenen) lateinischen Annalen zu, nicht den Text, den wir kennen. Als um $1420^{47}$ - Udo Arnold wagte die genaue Datierung auf „1422 (?)“48 - die Annalen ins Deutsche übertragen und bis 1419 fortgesetzt wurden, übersetzte der Bearbeiter die Intitulation seiner Vorlage wörtlich - wodurch sie uneindeutig wurde - und fügte einen Hinweis auf den Tod des Autors und die eigene deutsche Bearbeitung hinzu.

Dieser Redaktor ist anonym. Ich vermute einen Schreiber des um 1420 amtierenden Offizials. ${ }^{49}$ Dies war bis 1420 Johann von Reddin. Ab 1421 ist Nikolaus von Hohenstein durchgängig als pomesanischer Offizial bezeugt; eine neuerliche Amtsübergabe an Johann von Rehden um 1430 ist wenig glaubhaft. ${ }^{50}$

46 Strehlke konnte einige seiner amtlichen Handlungen nachweisen, etwa den Schiedsspruch über Besitzansprüche des ermländischen Domkapitels gegen den Deutschen Orden, worüber die Chronik selbst allerdings eher beiläufig und ohne Nennung der beteiligten Personen berichtet.

47 Wentas stärkstes Argument für Johann von Reddin als Gesamtautor ist, dass er 1420 noch als Urkundenzeuge auftrat und laut Strehlke - nach einer Funkstille von über 10 Jahren - am 19. Juli 1430 plötzlich wieder als Offizial des pomesanischen Bischofs testiert. Hier setzt Päsler, Sachliteratur (wie Anm. 39) S. 288 f. an: „Da die beiden Berliner Handschriften um 1420 entstanden sind, passe hier das noch syme tode nicht mehr“.

48 Arnold, Johann von Posilge (wie Anm. 39), Sp. 713, ohne Begründung.

49 Zumindest Johann von Reddin selbst hätte die Namensverhältnisse in der Intitulatio besser erklärt.

50 Eine einzelne Urkunde des Jahres 1430 nennt Johann von Rehden als Offizial. Strehlke fand sie als Nachtrag im sogenannten Kleinen Privilegienbuch des pomensanischen Domkapitels; vgl. Scriptores rerum Prussicarum (wie Anm. 8), Bd. 3, S. 31 f., Anm. 1; Textabdruck: Urkundenbuch zur Geschichte des vormaligen Bisthums Pomesanien, bearb. v. H. Cramer (Zeitschrift des Historischen Vereins für den Regierungsbezirk Marienwerder 15-17 (1885-1887), hier 16 (1886), S. 189 f., Nr. 130 (digital unter http://wwwl.uni-hamburg.de/Landesforschung/ 
Die Chronik ist folglich eher eine kollektive Leistung der Riesenburger Residenz als eine individuelle Schöpfung. Ausgerechnet die Teile, die sich von der schablonenhaften Form der Vorlagen freimachen ${ }^{51}$ - insbesondere fast die gesamte hintere Hälfte des Textes mit den Ereignissen im und nach dem Großen Krieg von 1410 - stammen nicht einmal im Entwurf von Johann von Posilge, sondern vom anonymen deutschen Bearbeiter und „Fortsetzer“. Der für diese Zeit hoch geschätzte Quellenwert der Chronik ist also nicht einem Offizial von Riesenburg zu danken, sondern dem anonymen deutschen Bearbeiter.

Dass er zum Kreis des Johanns von Reddin gehörte, bzw. dass die deutsche Chronik für die Kanzleiarbeit während seiner Amtszeit geschaffen wurde, ergibt sich aus den inneren Bezügen zwischen der Chronik und den Arbeitsschwerpunkten dieses Offizials. Johann von Reddin tritt häufiger als sein Vorgänger als Zeuge in pomesanischen Urkunden auf, und zwar bei Aktionen, die sich retrospektiv mit den kriegerischen Auseinandersetzungen befassen, die auch die deutschen Annalen beschreiben:

- 1412, als Bischof Johannes Mönch das Thorner Friedensinstrument von 1411 vidimiert; 52

- 1413, als Jagiełło und Witowt Samaiten erst nach ihrem Ableben dem Orden zugestehen wollen, und Heinrich von Plauen die Annahme dieser gegen den Frieden von Sallinwerder verstoßenden Urkunde ablehnt (JH 1769); schließlich

- 1419-1420 im Vorfeld des Schiedsspruchs, den der römische König Sigismund im Jahr 1420 fällte;

- August 1419 und Januar 1420, als Bischof Gerhard von Mewe auf Begehr des Hochmeisters sämtliche Urkunden vidimiert, die das Verhältnis des Ordensstaates zu Polen betrafen. Gerade hier ist der Offizial Johann von Reddin immer wieder als Zeuge eingesetzt worden. ${ }^{53}$

PomesanUB/4.189.html). Es handelt sich um die Erneuerung eines Mühlenprivilegs aus der Zeit des Johannes Ryman, als Johannes von Rehden sicher Offizial war. Ist der Zeugenname aus der ersten Urkunde in die Konfirmation übertragen worden?

51 Scriptores rerum Prussicarum (wie Anm. 8), Bd. 3, S. 38, läßt die Verwendung dieser stereotypen Vorlage mit dem Jahr 1393 enden. Der Herausgeber Strehlke vermutet ebd. S. 44 f., dass Annalista Thorunensis, Johann von Posilge und Dethmar auf einer gemeinsamen Quelle beruhen, die auch von Wigand von Marburg benutzt wurde. Detmar jedenfalls wird ab 1394 praktisch nicht mehr herangezogen.

52 Regesta historico-diplomatica Ordinis S. Mariae Theutonicorum 1198-1525, bearb. unter Mitwirkung zahlreicher Anderer von E. Joachim, hrsg. v. W. Hubatsch, Bd. 1, Göttingen 1948-1973; Bd. 2. Regesta privilegiorum Ordinis S. Mariae Theutonicorum. Regesten der Pergament-Urkunden aus der Zeit des Deutschen Ordens (im folgenden: JH), Göttingen 1948, Nr. 1751.

53 Vidimierungen mit Johann von Reddin als Zeugen: JH 1974 f. vom 9. August 1419; JH 1990 f., 1994 f., 1997-2000, fortgesetzt am 8. Januar 1420 (JH 2020, 2022). 
Eine derartige enge Verbindung zu den Themen der Annalen, dem mutmaßlichen Zeitpunkt der deutschen Bearbeitung und der politischen Arbeit eines Riesenburger Offizials haben wir sonst nicht, schon gar nicht bei Johann von Posilge.

Liest man die deutsche Übertragung und Fortschreibung auf den Vertrag von Sallinwerder von $1398^{54}$ hin bzw. auf seine Revision nach Witowts Aufstand und seine endgültige Aufhebung 1422 im Frieden von Melno-See, so bekommen die Notizen ein besonderes Gewicht. Die Bearbeitung gab den Ordensdiplomaten und den Gebietigern ein Dossier in die Hand, mit dem sie sich im militärischen und diplomatischen Dschungel der Litauenfahrten und der geschlossenen und gebrochenen Verträge zurechtfinden konnten. Die Chronik hat defensiven Charakter, scheint aber weniger in einem militärischen als in einem diplomatischen Kontext zu stehen (anders als die in ihr aufgegangene stereotype Vorlage).

\section{Die Kriegsziele Der Litauenfahrten}

Uns interessieren hier die Kriegsschilderungen. Sie sind in den ersten Jahrzehnten also in der von Johann von Posilge aufgrund älterer Annalen bearbeiteten Phase - eher stereotyp: Ermöglichte der Frost eine reyse $e^{55}$ oder verzögerte sich diese wegen zu mildem Wetter oder fand gar keine statt? Im Falle der reyse: wer erschlug wie viele Feinde, gab es bedeutende Tote (z. B. den Marschall Schindekopp im Jahr 1370) ${ }^{56}$, wie viele Gefangene machten die Ordenstruppen. ${ }^{57}$

Ein Beispiel. Für die Jahre 1377-1378 heißt es: Item uf den herbist quam herczog Albrecht von Osterrich ken Prusen mit II tusent pferdin und brachte mit ym vil grafen, herrin, ritter und knechte, und der homeister, her Wynrich von Knyprode, reysete mit ym ken Erogel und Pastow und die land dorumb. Kynstod wolde sie nicht obir Nerye ${ }^{58}$ losin und hatte die vorte alle vormachet. In desim jore wordin die Littowen sere obirgeretin und geswecht beyde von Prusen und Lyfland und von den Ungern. Anno domini 78 was der herczog von Lotringen und des pabist bruderson

54 Zum Frieden Scriptores rerum Prussicarum (wie Anm. 8), Bd. 3, S. 223 Anm. 2.

$55 \mathrm{Zu}$ den Litauenfahrten und ihrer allgemeinen Bedeutung für den Deutschen Orden vgl. Ekdahl, Christianisierung (wie Anm. 32), S. 191 (mit weiterführender Literatur).

56 Scriptores rerum Prussicarum (wie Anm. 8), Bd. 3, S. 91.

57 Zum Schicksal und zur ökonomischen Bedeutung der Kriegsgefangenen S. Ekdahl, The treatment of prisoners of war during the fighting between the Teutonic Order and Lithuania, in: Fighting for faith and caring for the sick, ed. M. Barber, (The Military Orders 1), London 1994, S. 263-269.

58 Neris, nördlicher Nebenfluß des Memel. 
Gregorii des elften ym lande czu Prusen; und dy herrin tatin mit yn eyne gute reyse bin ken Littowen und brochten VII' gefangen von dannen. ${ }^{59}$

So geht das jahrein jahraus. Es werden von beiden Seiten befestigte Plätze errichtet (meist Holzburgen), die dann vom Feind eingenommen und niedergebrannt werden - und beängstigend oft trifft es auch die festen Orte immer wieder, im Kulmerland etwa Rypin, Lipchin (Lipno) und Dobryn (Dobrzyń).$^{60}$ Die Verluste sind auf Seiten der Litauer hoch, aber auch die christlichen Kämpfer trifft es immer wieder hart. Von besonderem Interesse für den Annalisten ist die Zahl der jeweils gemachten Gefangenen, ${ }^{61}$ auch wenn es keinerlei Hinweise gibt, warum diese so genau verbucht werden.

Für den heutigen Leser ist die Sinnlosigkeit dieser Kämpfe erschreckend. Man begreift nicht, wie der doch als planvoller Administrator gerühmte Deutsche Orden sich in diese schier ewige Wiederkehr des Gleichen hineinverwickeln lassen konnte, deren Ausweglosigkeit ihm doch nach einigen Jahren hätte vor Augen stehen müssen. So wirkte das aber offenbar nicht auf die Zeitgenossen. Solange der Krieg auf litauischem Gebiet stattfand, war er für den Orden und für das Land Preußen ein positiver Wirtschaftsfaktor: Man profitierte vom Nimbus des Kreuzfahrerlandes, von den Plünderungen in Litauen und den Lösegeldzahlungen für die Gefangenen, von den Jahr für Jahr ins Land einreisenden Adligen mit ihrem Gefolge und vor allem von der Arbeitskraft der Kriegsgefangenen, für die kein Lösegeld aufgebracht wurde. Die Buchführung über die Gefangenenzahlen ist also keine Marotte des Chronisten, sondern die Dokumentation des wirtschaftlichen Gewinns, den der Feldzug dem Ordensland eingetragen hatte.

Um den damit verbundenen positiven Effekt für den Deutschen Orden zu unterlaufen, beauftragte Witowt 1407 eine Gesandtschaft zur Kurie, deren Ziel es war, das der pabist wolde gebin den applas und gnade furstin, herrin, rittern und knechtin, die durch got und der ere qwemen ken Littowin czu betwingen czu dem geloubin die Russin und heydin, dy den landen gelegin werin czu twingen, und uff die czu reysin glychir wys, als her gegebin ist dem Dutschin ordin czu Pruszin und Lyffland. ${ }^{62}$

Diese Anerkennung von Witowts Kriegen als Kreuzzug misslang, und so blieb allein dem Orden ein sakraler Aspekt seiner reysen. Und sie waren durchaus erfolgreich. Die allmähliche Verwandlung Samaitens in ein christlich dominiertes Territorium und die Durchdringung Litauens mit christlichen Würdenträgern

59 Scriptores rerum Prussicarum (wie Anm. 8), Bd. 3, S. 106 f.

60 Ebd., S. 301.

61 Hierzu grundklegend: Nikžentaitis, Prisoners of War (wie Anm. 17), S. 193-208.

62 Scriptores rerum Prussicarum (wie Anm. 8), Bd. 3, S. 288. 
gelang, aber der Orden konnte dieses Erfolges nicht froh werden, er leugnete sie sogar - weil sie der geistlichen Korporation das Recht auf Kriegsführung und das Recht auf Zwangsarbeit der Besiegten gefährdete. Bei der Belagerung der Marienburg 1410 wird der deutsche Fortsetzer nicht müde, die Belagerer als „Polen und Heiden" zu kennzeichnen - eine verwerfliche Allianz, die selbst diesen Krieg gegen ein christliches Königreich zum Heidenkrieg macht. Damit konnte der Orden auch über 1410 hinaus beim deutschen Adel die bindende Kraft nutzen, die die jährlichen Preußenfahrten mit ihren Ritterschlägen und Ehrentischen geschaffen hatten. So kann der deutsche Fortsetzer für 1414 vermelden: Dis grose jamir was irschollin yn Dutschin landin, das der ordin und das lant zcu Prusin geledin hattin von den Polen und den heydin, das vil guter ritter und knechte und etliche gebitiger von Dutschin landin quomen [worin = waren] bis ken Frankinfort, und kartin weder zcurocke, do sy irfurin, das deser krik was undernomen. Dys irschal ken Polen, und worin yn vorchte, das sy icht weder wordin obirretin. ${ }^{63}$

Selbst wenn hier eine gute Portion Wunschdenken am Werk war: in Preußen war sehr wohl bewusst, dass den Litauenfahrten eine den deutschen Adel bindende Kraft innewohnte, auf die man bauen konnte. Man muss von hier die deutschen Riesenburger Annalen gleichsam rückwärts lesen.

Der polnische König, klagt der deutsche Fortsetzer, führte im Jahr 1414 ein Heer von Litthowin, Walachin, Tattern, Rusin, Sameytin und vil uncristin, mit den sich nicht schemetin cristinliche furstin und herrin, ritter und knechte czu zcin off den ordin, der doch als eyne muer vil zeit was gewest vor dy cristinlant weder dy heydin, dy alle quomen mit dem konynge, ym czu helfin, und doch nicht sachin anders hattin weder den ordin, wend das sy dem konynge weldin helfin zcu syme rechtin, des her doch gar wenyng hatte. ${ }^{64}$

Die nüchterne Annalistik weicht einer propagandistischen Kampfschrift. Das ist der moralisch gefärbte Aufschrei des Unterlegenen. Natürlich führt der Orden einen reinen Verteidigungskrieg und zudem einen gerechten Krieg gegen all diese apokalyptischen Heidenscharen, die nun immer wieder Kirchen niederbrennen und sogar das Sakrament schänden. ${ }^{65}$ Die Richtung des Krieges und damit auch der Fluss des Kriegsprofits hat sich umgekehrt: Nunmehr ist Preußen Schauplatz der Kämpfe und der Verwüstungen. Damit kommen ältere Argumentationsmuster wieder zum Vorschein. Der Orden wird wieder zur Mauer, die die christlichen Länder vor den anbrandenden Heiden beschirmen soll. Das ist aus der Kreuzzugs-Propaganda des Mittelmeerraums übernommen, wo die asiatische

63 Ebd., S. 348.

64 Ebd., S. 340 f.

65 Ebd., S. 347. 
Bedrohung (die man nicht eben differenzierend alle als „Tartaren“ - Höllensöhne zusammenfasste) seit Tamerlans Angriff auf Damaskus gegenwärtig war.

Die Annalen schildern, ohne es zu thematisieren, die Zuspitzung der Lage: Verwüstest wird das Land Preußen, aber der Krieg gilt dem Deutschen Orden. ${ }^{66}$ Es sollte noch über einhundert Jahre dauern, bis der Hochmeister der Entscheidung zwischen dem Land und dem Orden nicht mehr auskam.

\section{UND GAB SIE IN DIE Ḧ̈NDE DER HEIDEN (Ps. 106,41)}

Das Scheitern Ulrichs von Jungingen bei Tannenberg beendete die Litauenfahrten und beraubte das Ordengebiet seiner bisherigen wirtschaftlichen Grundlage. Im Friede von Melno-See (1422) musste Paul von Rusdorf die neuen Verhältnisse anerkennen. Dagegen richtet sich, soweit sie den Orden selbst betrifft, die Ermahnung des Kartäusers, die dem Hochmeister 1428 übergeben wurde. ${ }^{67}$ Der Verfasser betont, er handle aus gantczer czugenegeter liebe und treue, die ich das Got weys, trage zcu deme erwirdigen orden und dem lande Preussen, ja sein Gewissen treibe ihn, seine Erkenntnisse in schrifften offenbar zu machen. Ein Ende der Ordensherrschaft kann sich der Kartäuser nunmehr vorstellen, nämlich falls mangels geistlicher - nicht etwa militärischer - Disziplin das Land durch Gottes Zorn widder bey dy heyden kome ${ }^{68}$ - wobei offen bleibt, wer hier mit „Heiden“ gemeint ist (Jagiełło?). Auch das warnende Beispiel der Templer wird beschworen, dy grosse lande hatten, ummb irer hofart willen und boses lebens hot men sy daraus getreben. ${ }^{69}$ Eine verwandte Synodalpredigt betrachtet Witowt alttestamentarisch als Vollstrecker der göttlichen Strafe. ${ }^{70}$ Diese Texte können auch als Kritik gegen das Pro-

66 Simon Grunau behauptet, daß die von ihm benutzte Handschrift die Verwüstung des Landes als Hauptthema in der Inskription nannte.

67 Die Ermahnung des Carthäusers, in: Scriptores rerum Prussicarum (wie Anm. 8), Bd. 4, hrsg. v. Th. Hirsch, Leipzig 1970, S. 448-465. Zur Schrift allgemein P. Oliński, Die Ermahnung des Kartäusers an die Deutschordensritter, in: Mittelalterliche Kultur und Literatur im Deutschordensstaat in Preußen. Leben und Nachleben (Sacra bella septentrionalia 1), hrsg. v. J. Wenta, S. Hartmann, G. Vollmann-Profe, Toruń 2008, S. 473-481; A. Mentzel-Reuters, Reformschrifttum und Humanismus. Der Deutsche Orden am Vorabend der Reformation, in: Die Rolle der Schriftlichkeit in den geistlichen Ritterorden des Mittelalters. Innere Organisation, Sozialstruktur, Politik (Ordines militares. Colloquia Torunensia Historica XV), hrsg. v. R. Czaja, J. Sarnowsky, Toruń 2009, S. 53-84, hier S. 62 f.

68 Ermahnung des Carthäusers (wie Anm. 66), S. 457.

69 Ebd., S. 457.

70 O. Günther, Eine Predigt vom preußischen Provinzialkonzil in Elbing 1427 und die „Ermahnung des Carthäusers“, Zeitschrift des Westpreussischen Geschichtsvereins 59 (1919), S. 69- 
gramm der "Schwertmission“ gelesen werden, wenn der Synodalprediger (der dem Deutschen Orden selbst angehört haben dürfte) Mt 9,3 zitiert: Misericordiam volo non sacrificium.

In dieser Zeit entstand ein neues historiographisches Standardwerk. Max Toeppen verlieh ihm den Namen Ältere Hochmeisterchronik, in den Handschriften gibt es keine einheitliche Bezeichnung. ${ }^{71}$ Das 1498 angelegte Exemplar aus der Bibliotheca Electoralis Friedrichs des Weisen (jetzt Jena Ms. El. philos. q. 2) ${ }^{72}$ nennt das Werk Cronica von Prewsen; der Codex Escorial K II 9 Fol. spricht von gescheffe und geschichte der brüder des hoen spitales sancte marie ... die geschen sein vil homeisters seliger mit den ungeleubigen in der heidenschaft auch im lande zu preussen mit litauen und thatern. ${ }^{73}$ Die ähnlich bezeichnete prewsche cronica des Königsberger Ordenshauses von 1432 (GÄB 32.23) scheint Nicolaus von Jeroschin zu sein; die ehemals Königsberger Handschrift 1558 (jetzt Biblioteka UMK, Toruń, rps 20/II) stammt aus der Residenz des samländischen Bischofs in Fischhausen. Doch stellt die Ältere Hochmeisterchronik ja eigentlich auch nichts anderes dar als eine gekürzte Prosafassung des Jeroschin, die bis zur Mitte des 15. Jahrhunderts fortgeführt wurde. Sie behält den asketischen Grundzug bei, indem sie z.B. von der Marienvision berichtet, die dem Bruder in Rehden zuteil wurde, der in einen strengeren Orden wechseln wollte. ${ }^{74}$

Die Darstellungsweise der Älteren Hochmeisterchronik wirkt auf uns angenehm positivistisch, da sie anscheinend nur Fakten gibt. Über Kriegsplanungen erfahren wir nichts, es hat eher den Eindruck, dass das Vorgehen von Versuch und Irrtum bestimmt war: Vor danczogen sy herende dy pomerische seyte und torsten keyn sloss me belegen, dorumme daz sy zcur Konitz nischt schuffen. Do sy vor Dirsaw dy stad quomen [...] [setzten sie zwei Speicher vor der Stadt in Brand - A. M.-R.]. Der wint stunt of dy stad, do von wart dy stad bornende. Do daz irsach das heer der vinde [...] sy ranten zcu rucke, und morten und slugen und vingen vil gutter lute. ${ }^{75}$

Die Schnittstelle zwischen der Bearbeitung der Chronik des Nikolaus von Jeroschin und den aktualisierenden Fortsetzungen bildet ein Lob Winrichs von

-111, hier S. 105 f. Zum Text vgl. Mentzel-Reuters, Reformschrifttum und Humanismus (wie Anm. 66), S. 63.

71 Zum Werk jetzt: M. Olivier, Geschichtsschreibung im mittelalterlichen Preußen und historiographischer Wissenstransder (13.-15. Jahrbundert), in: Mittelalterliche Eliten und Kulturfransfer östlich der Elbe. Interdisziplinäre Beiträge zu Archäologie und Geschichte im mittelalterlichen Ostmitteleuropa, hrsg. v. A. Klammt, S. Rossignol, Göttingen 2009, S. 151-168.

72 F. Pensel, Verzeichnis der altdeutschen und ausgewählter neuerer deutscher Handschriften in der Universitätsbibliothek Jena, Berlin 1986, S. $331 \mathrm{f}$.

73 Scriptores rerum Prussicarum (wie Anm. 8), Bd. 3, S. 521, Anm. 1.

74 Ebd., S. 546 f.

75 Ebd., S. 636. 
Kniprode. ${ }^{76}$ Es bricht aus dem nüchternen Erzählstil der restlichen Chronik aus und entwirft ein geschichtsphilosophisches Tableau auf der Grundlage der Psalmen 105-106. Der bereits zitierte Ps. 105 gibt die Folie für die Landnahme und Unterwerfung, wo nicht Versklavung, der Einwohner Ps 106 hingegen bedroht Frevler mit dem Verlust des Landes.

Do dy selben alden herren des ordens lebeten, sy hilden veste dy gebot gotis, und warn yn seiner libe heis. Dorumme wurden sy segeh afft ken al iren vinden. Aber dornoch leider begunden sy ap zcu nehmen in seyner libe, und worden kallt und von tag zcu tage (leider sey is gote geclaget und seyner mutter) yo kelder an rechtem leben. Sy irkanten nicht noch leider irkennen wie ire vorfarn mildiclich vorgussen ir blut, und in den tod giengen kegen die heiden umb des globen und der gerechtikeit willen und wie das got en gegeben hat Prewserlant und ouch Leifland wunderlich zcu besitczen bass uff diszen tag, und die inwoner diszer beiden lande in zcu gebote mussen steen. Dorumme vorhenget got vele phlogen obir disze armen lande ..., uff das sie sich irkennen, doran sie sich nicht keren und suchen nort eyn itczlicher, was em nütcze ist, und nicht den nutcz gotis des hern Jhesu Cristi zcu irer zelen selikeit. Dorumme leiden sy nicht alleyene von den heiden, szunder ouch von etczlichen cristen fursten [...]. ${ }^{77}$

Die Mystifikation der „alten“ Ordenkämpen zu Marienrittern hinterlässt hier ihre Spuren. Dass die inwoner diszer beiden lande in zcu gebote mussen stehen, ist wörtlich aus Ps. 105,44 übernommen (et laborespopulorum possederunt). Ps 106,39 f. wird in den folgenden Zeilen ausgeschrieben. Der Gedanke, dass Gott seinen Auserwählten das Gelobte Land auch wieder fortnehmen kann, ist ein geläufiger biblischer Topos. ${ }^{78}$

Hier hat er neue Aktualität, da der militärische Misserfolg als Strafe für ein sündiges Leben begriffen wird.

Diese moralisierende Haltung bestimmt freilich nicht die Berichterstattung der Chronik. Sie ähnelt in der Schilder des Auf und Ab der Kämpfe von Jahr zu Jahr eher der Posilge-Chronik, so dass man vermuten könnte, der zitierten Passage liege eine Ermahnungsrede aus einem Ordenskapitel zugrunde.

\section{ZUSAMMENFASSUNG}

Einen Kriegszweck im Sinne der eingangs zitierten Definition von Clausewitz können wir tatsächlich benennen, obschon er dem Militärtheoretiker abstrus

\footnotetext{
76 Ebd., S. 599-601.

77 Ebd., S. 601.

78 Lev 26,30, Richter 2,14, Ps 78,59, Hes 23,18 u. ö.
} 
erschienen wäre: Die Kriegsberichte der Deutschordenschroniken heben auf eine typologische Übereignung der preußischen und livländischen Territorien als dem Orden bestimmte "terra promissa“ ab, die er dem christlichen Glauben zuzuführen und gegen heidnische Überfälle zu verteidigen hat. Eine Ablösung der Terra vom Orden ist darum undenkbar. Das Ideal des spirituell-asketisch überhöhten Ordenskriegers, der sich auch im Kampf der Tugenden gegen Satan auszeichnet, wie es bei Peter von Dusburg massiv vertreten wird, schwächt sich dabei im Laufe des 14. Jahrhunderts ab und kehrt sich am Ende - d. h. spätestens nach 1411 - gegen den Orden. Das Ausbleiben eines durchschlagenden Erfolges gegen die polnisch-litauische Union wird als spirituelles Versagen interpretiert. Die ursächlichen Zusammenhänge zwischen militärischen Operationen und wirtschaftlichem Wohlergehen der „Blütezeit“ vor 1410 oder der seit dem Ersten Thorner Frieden 1414 beklagten „Verderbnis der preußischen Lande“ können die Chroniken hingegen nicht adäquat thematisieren. Darum verschwimmen auch die aktuellen Kriegsziele jeweils im Nebel der Erinnerungen. 
